\title{
Performance Analysis on MIMO-OFCDM Systems with Multi-Code Transmission
}

\author{
Yiqing Zhou, Member, IEEE, and Tung-Sang Ng, Fellow, IEEE
}

\begin{abstract}
This letter proposes an analytical approach to evaluate the performance of MIMO-OFCDM systems [1] with multicode transmission. Assuming zero-forcing successive interference cancellation (ZF-SIC) in the space domain and MMSE detection in the frequency domain, it is shown that at each step of SIC, the error events on multiple code channels are correlated to each other, which make the performance evaluation difficult due to the involvement of a complicated multivariate probability. By approximating the multivariate probability by a series of two-variate probabilities, the proposed analytical approach takes the correlation into account and provides accurate performance estimations. The analytical results are verified by simulations and shown to be more accurate than those where no correlation is considered.
\end{abstract}

Index Terms-OFCDM, two-dimensional spreading, multicode, MIMO, interference cancellation.

\section{INTRODUCTION}

$\mathbf{O}$ RTHOGONAL frequency and code division multiplexing (OFCDM) has been shown to be a promising candidate for the downlink transmission in future high speed wireless communications [2]. Based on OFDM, OFCDM employs two-dimensional (2-D) spreading, where each data symbol is spread in the time domain with $N_{T}$ chips and in the frequency domain with $N_{F}$ chips. To increase data rate, multi-code transmission can be employed, where multiple 2-D codes are assigned to one single user. By means of simulations, OFCDM has been investigated in multiple input multiple output (MIMO) systems on various topics like near-far problems [3], sub-optimum maximum likelihood detection [4], joint iterative detection and optimal power allocation [1]. In this letter, an analytical study is carried out on the uncoded MIMOOFCDM with zero-forcing successive interference cancellation (ZF-SIC) and imperfect channel estimation.

The main challenge for the performance analysis is to accurately estimate the error propagation in SIC. A single carrier MIMO (SC-MIMO) system with ZF-SIC has been analytically investigated in [5]. At each step of SIC, since one data symbol was transmitted on each antenna in SC-MIMO, only one error event would occur. However, in the multicode MIMO-OFCDM system, each antenna sends multiple data symbols (one symbol on each code channel) at the same time. So more than one data decision error could occur, and

Manuscript received August 23, 2008; revised December 28, 2008, March 30, 2009, and May 24, 2009; accepted May 26, 2009. The associate editor coordinating the review of this letter and approving it for publication was Y.-C. Ko.

The authors are with the Department of Electrical and Electronic Engineering, University of Hong Kong, Pokfulam Road, Hong Kong (e-mail: \{yqzhou, tsng\}@eee.hku.hk).

Digital Object Identifier 10.1109/TWC.2009.081136 these errors are correlated to each other. Thus, the performance analysis involves a multivariate Gaussian distribution, which becomes intractable when the number of code channels increases. Although the BER analysis of IC technologies has been studied extensively [6][7], the problem is only partly solved for systems with few stages and users/code channels, e.g., two-stage and two users in [7], due to the complex multivariate distribution. The proposed analytical approach approximates the multivariate distribution with a series of two events distributions, thus reduces the analysis complexity dramatically and takes the error event correlation into account as well. This approach can also be applied to other IC schemes where error event correlation must be considered. It will be shown that the analytical results obtained from the proposed approach are close to simulations and more accurate than those where no error correlation is considered.

In the rest of this letter, Section II gives a brief introduction on the system model. The performance of MIMO-OFCDM is analytically evaluated in Section III. Numerical and simulation results are presented in Section IV to verify the analytical results. Finally, conclusions are drawn in the last section. In the following, bold capital letters stand for matrices and normal letters are for other scalars.

\section{SySTEM MOdeL}

\section{A. Transmitter}

A brief introduction is given in this letter on the system model. Readers could refer to [1] for a detailed description. In MIMO-OFCDM, independent bit streams are transmitted at $n_{t}$ antennas. At each antenna, the stream is serial to parallel converted into $K$ streams, corresponding to $K$ data code channels. On each code channel, information bits are modulated and 2-D spread. Signals from all $K$ data code channels are then added up at the code multiplexer, and blockinterleaved in the frequency domain, so that the $N_{F}$ subcarriers carrying the same data symbol are separated in the frequency band, providing large frequency diversity gain. At the same time, known pilot symbols are employed on each sub-carrier and spread in the time domain. Different orthogonal spreading codes are used by the pilot channel of different antennas. Using packet transmission, each packet starts with $N_{P}$ OFCDM symbols for pilot, followed by $N_{D}$ symbols for data. The signal transmitted during the $i^{\text {th }}$ OFCDM symbol on the $m^{\text {th }}$ sub-carrier at the $p^{\text {th }}$ antenna is given by

$s_{p, m, i}=\left\{\begin{array}{l}d_{p i l o t, p, m} c_{N_{P}, i}^{(p)}, \quad 0 \leq i \leq N_{P}-1 \\ \sum_{k=0}^{K-1} d_{p, i, m, k} C_{i, m, k}, N_{P} \leq i \leq N_{P}+N_{D}-1\end{array}\right.$ 


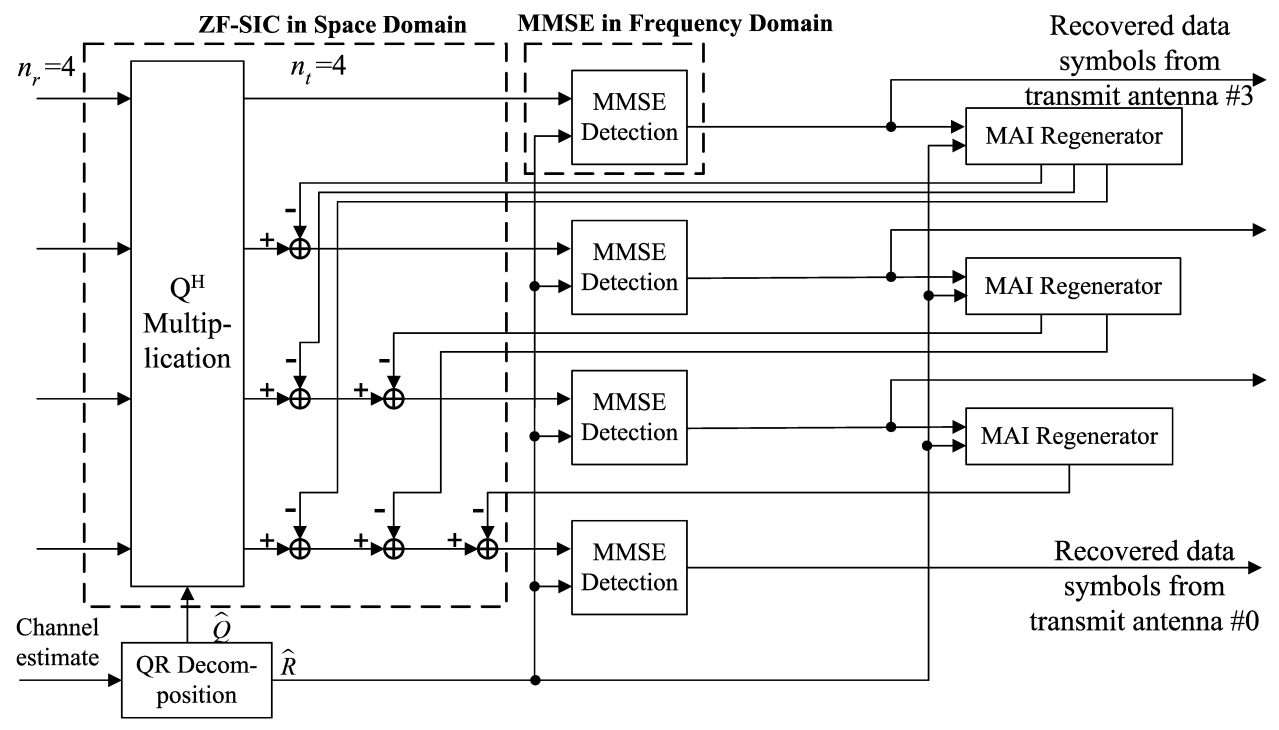

Fig. 1. Example of ZF-MMSE-SIC signal detection for $n_{t}=n_{r}=4$

$$
\widetilde{h}_{\bar{q}, \bar{p}, \bar{m}}=\frac{1}{d_{\text {pilot }, \bar{p}, \bar{m}}}\left(\sum_{p=0}^{n_{t}-1} h_{\bar{q}, p, \bar{m}} d_{\text {pilot }, p, \bar{m}}\left(\frac{1}{N_{P}} \sum_{\bar{i}=0}^{N_{P}-1} c_{N_{P}, \bar{i}}^{(p)} c_{N_{P}, \bar{i}}^{(\bar{p})}\right)+\frac{1}{N_{P}} \sum_{\bar{i}=0}^{N_{P}-1} \eta_{\bar{q}, \bar{i}, \bar{m}} c_{N_{P}, \bar{i}}^{(\bar{p})}\right)=h_{\bar{q}, \bar{p}, \bar{m}}+\nu_{\bar{q}, \bar{p}, \bar{m}}
$$

where $d_{\text {pilot,p,m }}$ is the pilot symbol with $E\left\{\left|d_{\text {pilot }, p, m}\right|^{2}\right\}=$ $\beta P_{d}, d_{p, i, m, k}$ is the data symbol of the $k^{\text {th }}$ code channel with $E\left\{\left|d_{p, i, m, k}\right|^{2}\right\}=P_{d}$, and $c_{N_{P}, i}^{(p)}$ and $C_{i, m, k}$ are the spreading code for the pilot and data channel, respectively.

A frequency selective and slow fading channel is adopted. Let $h_{q, p, m}$ denote the complex channel fading on the $m^{\text {th }}$ sub-carrier from the $p^{\text {th }}$ transmit antenna to the $q^{\text {th }}$ receive antenna, whose amplitude and phase are Rayleigh distributed with $P_{c h}=E\left\{\left|h_{q, p, m}\right|^{2}\right\}$ and uniformly distributed in $[0,2 \pi)$, respectively. Note that in slow fading channels, the orthogonality in the time domain can be kept among the 2-D spread multi-code channels and the spreading in the time domain has no influence on the system performance. However, 2-D spreading is still employed instead of the one dimensional spreading in the frequency domain to make the system compatible to other OFCDM systems and suitable for future research in fast fading channels.

\section{B. Receiver}

At each receive antenna, signals are processed by the matched filter, FFT block, deinterleaver and demultiplexer. On the $\bar{m}^{\text {th }}$ sub-carrier, the pilot signals are despread in the time domain, after which the channel factor from the $\bar{p}^{\text {th }}$ transmit antenna to the $\bar{q}^{\text {th }}\left(0 \leq \bar{q} \leq n_{r}-1\right)$ receive antenna can be obtained as (2), where $\eta_{\bar{q}, \bar{i}, \bar{m}}$ is the zero-mean background noise with a variance of $\sigma_{n}^{2} \cdot \eta_{\bar{q}, \bar{i}, \bar{m}}$ is independent to each other for different sub-carriers, antennas and OFCDM symbol durations. Given QPSK modulated pilot symbols, the variance of $\nu_{\bar{q}}, \bar{p}, \bar{m}$ is $\sigma_{H}^{2}=\sigma_{n}^{2} /\left(\beta P_{d} N_{P}\right)$. Meanwhile, assuming $\left\{C_{N_{T}}^{(0)}, C_{N_{F}}^{(\bar{k})}\right\}$ as the desired code channel, after time domain despreading, the data signal on the $\bar{m}^{\text {th }}$ subcarrier of the $\bar{q}^{\text {th }}$ receive antenna is given by

$$
\begin{aligned}
z_{\bar{q}, \bar{m}}= & h_{\bar{q}, \bar{p}, \bar{m}}\left(d_{\bar{p}, \bar{k}} c_{N_{F}, \bar{m}}^{(\bar{k})}\right) \\
& +\underbrace{\sum_{\substack{p=0 \\
p \neq \bar{p}}}^{n_{t}-1} h_{\bar{q}, p, \bar{m}}\left(\sum_{k=0}^{K_{C}} d_{p, k} c_{N_{F}, \bar{m}}^{(k)}\right)}_{M A I} \\
& +\underbrace{h_{\bar{q}, \bar{p}, \bar{m}}\left(\sum_{\substack{k=0 \\
k \neq \bar{k}}}^{K_{C}} d_{\bar{p}, k} c_{N_{F}, \bar{m}}^{(k)}\right)}_{M C I}+\varepsilon_{\bar{q}, \bar{m}}
\end{aligned}
$$

where $K_{C}$ is the number of codes in the interfering code set $\Omega_{F}=\left\{\left\{C_{N_{T}}^{(0)}, C_{N_{F}}^{\left(k_{F}\right)}\right\} \mid k_{F} \neq \bar{k}\right\}$, MAI is the interference from other transmit antennas, MCI is the interference from signals radiated by the same antenna but on different code channels in $\Omega_{F}$, and $\varepsilon_{\bar{q}}, \bar{m}$ is the noise with zero mean and a variance of $\sigma_{n}^{2} / N_{T}$.

Thus, ZF-MMSE-SIC detection is employed with ZF-SIC in the space domain to combat MAI and the MMSE detection in the frequency domain to suppress MCI. As illustrated in Fig. 1 , based on QR decomposition, ZF-SIC is carried out first. Let $\widehat{\mathrm{H}}_{\bar{m}}$ represent the $n_{r} \times n_{t}$ estimated channel matrix on the $\bar{m}^{\text {th }}$ sub-carrier. The QR decomposition of $\widehat{\mathrm{H}}_{\bar{m}}$ is given by $\widehat{\mathrm{H}}_{\bar{m}}=\widehat{\mathrm{Q}}_{\bar{m}} \widehat{\mathrm{R}}_{\bar{m}}$, where $\widehat{\mathrm{Q}}_{\bar{m}}$ is a $n_{r} \times n_{t}$ orthogonal matrix and $\widehat{\mathrm{R}}_{\bar{m}}$ is a $n_{t} \times n_{t}$ upper triangular matrix. After multiplying the received signal vector with $\widehat{\mathrm{Q}} \frac{H}{m}$, the last element or the $\left(n_{t}-1\right)^{\text {th }}$ element of the resultant vector is free of MAI and 


$$
\begin{aligned}
& y_{n_{t}-1-s, \bar{k}}=\lambda_{s}\left(\sum_{\bar{m}} l_{s, \bar{m}} \cdot \gamma_{n_{t}-1-s, \bar{m}}^{(s)}\right)=d_{n_{t}-1-s, \bar{k}}+\underbrace{\sum_{\bar{m}} \omega_{s, \bar{m}} c_{N_{F}, \bar{m}}^{(\bar{k})}\left[\sum_{p=n_{t}-s}^{n_{t}-1} \widehat{r}_{\bar{m}, n_{t}-1-s, p} \sum_{k=0}^{K_{C}}\left(d_{p, k}-\widehat{d}_{p, k}\right) c_{N_{F}, \bar{m}}^{(k)}\right]}_{M A I_{n_{t}-1-s, \bar{k}}} \\
& +\underbrace{\lambda_{s} \sum_{\bar{m}} \sum_{k=0, k \neq \bar{k}}^{K_{C}} \omega_{s, \bar{m}} c_{N_{F}, \bar{m}}^{(\bar{k})} \widehat{r}_{\bar{m}, n_{t}-1-s, n_{t}-1-s} d_{n_{t}-1-s, k} c_{N_{F}, \bar{m}}^{(k)}}_{M C I_{n_{t}-1-s, \bar{k}}}+\underbrace{\lambda_{s} \sum_{\bar{m}} \omega_{s, \bar{m}} c_{N_{F}, \bar{m}}^{(\bar{k})} \eta_{n_{t}-1-s, \bar{m}}}_{\text {oi }_{n_{t}-1-s, \bar{k}}}
\end{aligned}
$$

provides an estimation for the multi-code signal $\sum_{k=0}^{K_{C}} d_{n_{t}-1, k}$ transmitted from the $\left(n_{t}-1\right)^{\text {th }}$ antenna. Since $\sum_{k=0}^{\substack{k=0 \\ K_{C}}} d_{n_{t}-1, k}$ are impressed on $N_{F}$ interleaved sub-carriers with $\bar{m}=0$, $M / N_{F}, \cdots, M-M / N_{F}$ ( $M$ is the total number of subcarriers), MMSE detection is employed in the frequency domain to recover each data symbol, which is needed for the MAI cancellation at the next step. In general, the data transmitted from the $\left(n_{t}-1-s\right)^{\text {th }}$ antenna can be obtained after the $s^{\text {th }}$ step of the ZF-MMSE-SIC detection. When $s=0$, there is no MAI cancellation. When $s>0$, after the MAI cancellation, the resultant signal for the $\left(n_{t}-1-s\right)^{\text {th }}$ transmit antenna is given by

$$
\begin{aligned}
& \gamma_{n_{t}-1-s, \bar{m}}^{(s)} \\
& =\gamma_{n_{t}-1-s, \bar{m}}-\left(\sum_{p=n_{t}-s}^{n_{t}-1} \widehat{r}_{\bar{m}, n_{t}-1-s, p} \sum_{k=0}^{K_{C}} \widehat{d}_{p, k} c_{N_{F}, \bar{m}}^{(k)}\right) \\
& =\widehat{r}_{\bar{m}, n_{t}-1-s, n_{t}-1-s} \sum_{k=0}^{K_{C}} d_{n_{t}-1-s, k} c_{N_{F}, \bar{m}}^{(k)}+\eta_{n_{t}-1-s, \bar{m}} \\
& +\underbrace{\sum_{p=n_{t}-s}^{n_{t}-1} \widehat{r}_{\bar{m}, n_{t}-1-s, p} \sum_{k=0}^{K_{C}}\left(d_{p, k}-\widehat{d}_{p, k}\right) c_{N_{F}, \bar{m}}^{(k)}}_{M A I_{n_{t}-1-s, \bar{m}}},
\end{aligned}
$$

where $\gamma_{n_{t}-1-s, \bar{m}}$ is the $\left(n_{t}-1-s\right)^{\text {th }}$ output of $\widehat{\mathrm{Q}} \frac{H}{m}$ multiplication, $\widehat{r}_{\bar{m}, i, j}$ is the $(i, j)^{\text {th }}$ element of $\widehat{\mathrm{R}}_{\bar{m}}, \widehat{d}_{p, k}$ is the recovered data symbol, $M A I_{n_{t}-1-s, \bar{m}}$ is the residual MAI, and $\eta_{n_{t}-1-s, \bar{m}}$ is the zero-mean noise with a variance of $\sigma^{2}=\left(K_{C}+1\right) P_{d} n_{t} \sigma_{H}^{2}+\sigma_{n}^{2} / N_{T}$ [1]. Then, MMSE detection is employed to recover $d_{n_{t}-1-s, \bar{k}}$ from $\gamma_{n_{t}-1-s, \bar{m}}^{(s)}$. Generally, it is difficult to get a close-form expression for the MMSE weights due to the involvement of matrix inversion. However, assuming full load, i.e., $K=N$, a simplified MMSE weight can be obtained [8]

$$
\begin{aligned}
& l_{s, \bar{m}, \bar{k}}=\frac{E\left\{d_{n_{t}-1-s, \bar{k}} \cdot\left(\gamma_{n_{t}-1-s, \bar{m}}^{(s)}\right)^{*} \mid \widehat{r}_{\bar{m}, n_{t}-1-s, n_{t}-1-s}\right\}}{E\left\{\left|\gamma_{n_{t}-1-s, \bar{m}}^{(s)}\right|^{2} \mid \widehat{r}_{\bar{m}, n_{t}-1-s, n_{t}-1-s}\right\}} \\
& =\frac{P_{d} \cdot \widehat{r}_{\bar{m}, n_{t}-1-s, n_{t}-1-s} c_{N_{F}, \bar{m}}^{(\bar{k})}}{P_{d}\left(K_{C}+1\right) \widehat{r}_{m, n_{t}-1-s, n_{t}-1-s}^{2}+E\left\{\left|M A I_{n_{t}-1-s, \bar{m}}\right|^{2}\right\}+\sigma^{2}} \\
& =\omega_{s, \bar{m}} c_{N_{F}, \bar{m}}^{(\bar{k})},
\end{aligned}
$$

where $E\left\{\left|M A I_{n_{t}-1-s, \bar{m}}\right|^{2}\right\}$ depends on the data decisions obtained from the $0^{\text {th }}$ to the $(s-1)^{\text {th }}$ step, given by

$$
\begin{aligned}
& E\left\{\left|M A I_{n_{t}-1-s, \bar{m}}\right|^{2} \mid\left\{N_{e, 0}, \cdots, N_{e, s-1}\right\}\right\} \\
& =2 P_{d} \sum_{p=n_{t}-s}^{n_{t}-1}\left|\widehat{r}_{\bar{m}, n_{t}-1-s, p}\right|^{2} N_{e, n_{t}-1-p}
\end{aligned}
$$

where QPSK modulation is assumed, $N_{e, p}$ is the number of bit errors at the $p^{\text {th }}$ step, and bit errors at the real and imaginary parts are independent to each other. The output of MMSE detection is further normalized by $\lambda_{s}=$ $\left(\sum_{\bar{m}} \omega_{s, \bar{m}} \widehat{r}_{\bar{m}, n_{t}-1-s, n_{t}-1-s}\right)^{-1}$ to simplify the expressions in the next section. Thus, the decision variable for the data on the $\bar{k}^{\text {th }}$ code channel of the $s^{\text {th }}$ transmit antenna is obtained as (7).

In this letter, ZF-based schemes are considered in both channel estimation and signal detection in the space domain, due to their simplicity and acceptable performance. MMSE-based schemes can also be employed, providing better performance at the cost of extra complexity. Since the main idea of the proposed analytical approach keeps the same for either ZFor MMSE-based schemes, ZF-based ones are employed to simplify the derivations in the next section. Moreover, it has been shown that with multi-code transmission, the MIMOOFCDM suffers from MAI and MCI, and ZF-MMSE-SIC is needed to combat these interferences. To reduce the transceiver complexity, an alternative architecture, i.e., transceiver B, can be considered, where the data at each transmit antenna is spread only in the time domain with an antenna-specific orthogonal code of length $N_{T, B}\left(N_{T, B} \geq n_{t}\right)$ and single code transmission is assumed. Hence, at each receive antenna, using time domain despreading, data signals transmitted from different antennas could be easily separated. Then maximum ratio combining (MRC) is employed to collect the transmitted data from different receive antennas and a full receive diversity of order $n_{r}$ is achieved. Since there is no need to detect signals in the space domain, receiver complexity is greatly reduced. However, the MIMO-OFCDM with transceiver B can only achieve $1 / N_{T, B}$ data rate as high as that provided by the transceiver A employed in this letter where multi-code transmission and ZF-MMSE-SIC detection are considered.

\section{PERFORMANCE ANALYSIS}

\section{A. BER at the $0^{\text {th }}$ Step}

With QPSK modulation, the complex data symbol on the $\bar{k}^{\text {th }}$ code channel of the $\left(n_{t}-1\right)^{\text {th }}$ antenna can be written as $d_{n_{t}-1, \bar{k}}=\sqrt{P_{d} / 2}\left(d_{I, n_{t}-1, \bar{k}}+j \cdot d_{Q, n_{t}-1, \bar{k}}\right)$. Assuming 


$$
\begin{aligned}
& P_{e, \widehat{\mathrm{H}_{\mathrm{F}}}}^{(s)}=\operatorname{Pr}\left(\widehat{d}_{I, n_{t}-1-s, \bar{k}} \neq d_{I, n_{t}-1-s, \bar{k}} \mid \widehat{\mathrm{H}}_{F}\right)=\sum_{N_{e, 0}=0}^{2 K_{c}+2} \cdots \sum_{N_{e, s-1}=0}^{2 K_{c}+2} P_{e, \widehat{\mathrm{H}}_{F},\left\{N_{e, 0}, \cdots, N_{e, s-1}\right\}}^{(s)} \operatorname{Pr}\left(N_{e, 0}, \cdots, N_{e, s-1} \mid \widehat{\mathrm{H}}_{F}\right)(10) \\
& P_{e, \widehat{\mathrm{H}}_{F},\left\{N_{e, 0}, \cdots, N_{e, s-1}\right\}}^{(s)}=\operatorname{Pr}\left(\widehat{d}_{I, n_{t}-1-s, \bar{k}} \neq d_{I, n_{t}-1-s, \bar{k}}\left|\widehat{\mathrm{H}}_{F},\left\{N_{e, 0}, \cdots, N_{e, s-1}\right\}\right|\right)=Q\left(\sqrt{P_{d} /\left(2 \sigma_{I, \bar{k}}^{2}(s)\right)}\right) .
\end{aligned}
$$

that there is no phase imbalance at the receiver, the BER of the real and imaginary parts of the decision variable $y_{n_{t}-1, \bar{k}}$ should be the same. Moreover, suppose that the data on different code channels and the background noises on different sub-carriers are independent to each other. Given the estimated channel matrix on $N_{F}$ sub-carriers $\widehat{\mathrm{H}}_{F}=$ $\left\{\widehat{\mathrm{H}}_{0}, \widehat{\mathrm{H}}_{M / N_{F}}, \cdots, \widehat{\mathrm{H}}_{M-M / N_{F}}\right\}$, the variances of the MCI and noise in the real part of $y_{n_{t}-1, \bar{k}}$ (see (7)), i.e., $y_{I, n_{t}-1, \bar{k}}$, are given by

$$
\left\{\begin{array}{l}
\sigma_{I, M C I, \bar{k}}^{2}(0) \\
=\frac{P_{d}}{2} \lambda_{0}^{2} \sum_{k=0, k \neq \bar{k}}^{K_{C}}\left|\sum_{\bar{m}} \omega_{0, \bar{m}} \widehat{r}_{\bar{m}, n_{t}-1, n_{t}-1} c_{N_{F}, \bar{m}}^{(k)} c_{N_{F}, \bar{m}}^{(\bar{k})}\right|^{2} \\
\sigma_{I, N o i, \bar{k}}^{2}(0)=\frac{\sigma^{2}}{2} \lambda_{0}^{2} \sum_{\bar{m}}\left|\omega_{0, \bar{m}}\right|^{2}
\end{array}\right.
$$

Since MCI can be accurately approximated as a Gaussian variable in MMSE detection, the sum of the MCI and noise can be modeled as a zero-mean Gaussian noise with a variance of $\sigma_{I}^{2}(0)=\sigma_{I, M C I, \bar{k}}^{2}(0)+\sigma_{I, N o i, \bar{k}}^{2}(0)$. Thus, the conditional BER at the $0^{\text {th }}$ step of ZF-MMSE-SIC detection is given by

$$
\begin{aligned}
P_{e, \widehat{\mathrm{H}}_{F}}^{(0)} & =\operatorname{Pr}\left(\widehat{d}_{I, n_{t}-1, \bar{k}} \neq d_{I, n_{t}-1, \bar{k}} \mid \widehat{\mathrm{H}}_{F}\right) \\
& =Q\left(\sqrt{P_{d} /\left(2 \sigma_{I}^{2}(0)\right)}\right)
\end{aligned}
$$

where $Q(x)=1 / \sqrt{2 \pi} \int_{x}^{+\infty} e^{-t^{2} / 2} d t$.

\section{B. BER at the $s^{\text {th }}$ Step}

Let $\operatorname{Pr}\left(N_{e, 0}, \cdots, N_{e, s-1} \mid \widehat{\mathrm{H}}_{F}\right)$ denote the probability of the event that there are $N_{e, 0}, \cdots, N_{e, s-1}$ bit errors at the $0^{\text {th }}, \cdots,(s-1)^{\text {th }}$ step, respectively. The conditional BER at the $s^{\text {th }}$ step of the detection can be expressed as (10), where $2 K_{C}+2$ is the maximum number of bit errors at each step, and $P_{e, \widehat{\mathrm{H}}_{F},\left\{N_{e, 0}, \cdots, N_{e, s-1}\right\}}$ is the BER conditioned on both channel information and error event $\left\{N_{e, 0}, \cdots, N_{e, s-1}\right\}$. First of all, $P_{e, \widehat{\mathrm{H}}_{F},\left\{N_{e, 0}, \cdots, N_{e s-1}\right\}}^{(s)}$ can be obtained by taking $M A I_{n_{t}-1-s, \bar{k}}$ as a Gaussian distributed variable. The variance of the real part of $M A I_{n_{t}-1-s, \bar{k}}$ is given by

$$
\begin{aligned}
& \sigma_{I, M A I, \bar{k}}^{2}\left(s,\left\{N_{e, 0}, \cdots, N_{e, s-1}\right\}\right) \\
& =E\left\{\left(M A I_{I, n_{t}-1-s, \bar{k}}\right)^{2} \mid\left\{N_{e, 0}, \cdots, N_{e, s-1}\right\}\right\} \\
& \approx P_{d} \lambda_{s}^{2} \sum_{\bar{m}}\left|\omega_{s, \bar{m}}\right|^{2} \sum_{p=n_{t}-s}^{n_{t}-1}\left|\widehat{r}_{\bar{m}, n_{t}-1-s, p}\right|^{2} N_{e, n_{t}-1-p},
\end{aligned}
$$

where the correlation of $M A I_{n_{t}-1-s, \bar{k}}$ on different subcarriers is ignored. Assuming that the real part of MAI, MCI and noise are independent to each other, their sum can be taken as a zero-mean Gaussian noise with a variance of $\sigma_{I, \bar{k}}^{2}(s)=\sigma_{I, M A I, \bar{k}}^{2}\left(s,\left\{N_{e, 0}, \cdots, N_{e, s-1}\right\}\right)+\sigma_{I, M C I, \bar{k}}^{2}(s)+$
$\sigma_{I, N o i, \bar{k}}^{2}(s)$, where $\sigma_{I, M C I, \bar{k}}^{2}(s)$ and $\sigma_{I, N o i, \bar{k}}^{2}(s)$ can be obtained by replacing $\lambda_{0}, \omega_{0, \bar{m}}$ and $\widehat{r}_{\bar{m}, n_{t}-1, n_{t}-1}$ in (8) by $\lambda_{s}, \omega_{s, \bar{m}}$ and $\widehat{r}_{\bar{m}, n_{t}-1-s, n_{t}-1-s}$, respectively. Thus, $P_{e, \widehat{\mathrm{H}}_{F},\left\{N_{e, 0}, \cdots, N_{e, s-1}\right\}}^{(s)}$ is given by (12).

Next, $\operatorname{Pr}\left(N_{e, 0}, \cdots, N_{e, s-1} \mid \widehat{\mathrm{H}}_{F}\right)$ can be rewritten in an iterative way as follows

$$
\begin{aligned}
\operatorname{Pr}( & \left.N_{e, 0}, \cdots, N_{e, s-1} \mid \widehat{\mathrm{H}}_{F}\right) \\
=\operatorname{Pr} & \left(N_{e, s-1} \mid N_{e, 0}, \cdots, N_{e, s-2}, \widehat{\mathrm{H}}_{F}\right) \\
& \cdot \operatorname{Pr}\left(N_{e, s-2} \mid N_{e, 0}, \cdots, N_{e, s-3}, \widehat{\mathrm{H}}_{F}\right) \\
& \quad \cdots \operatorname{Pr}\left(N_{e, 1} \mid N_{e, 0}, \widehat{\mathrm{H}}_{F}\right) \cdot \operatorname{Pr}\left(N_{e, 0} \mid \widehat{\mathrm{H}}_{F}\right) .
\end{aligned}
$$

To calculate (13), $\operatorname{Pr}\left(N_{e, 0} \mid \widehat{\mathrm{H}}_{F}\right)$ should be obtained first. Define the decision error on the $k^{\text {th }}$ data code channel at the $0^{\text {th }}$ step as

$$
\begin{aligned}
e_{k}^{(0)} & =e_{I, k}^{(0)}+j \cdot e_{Q, k}^{(0)} \\
& =1 / 2\left(\begin{array}{l}
\left|d_{I, n_{t}-1, k}-\widehat{d}_{I, n_{t}-1, k}\right| \\
+j\left|d_{Q, n_{t}-1, k}-\widehat{d}_{Q, n_{t}-1, k}\right|
\end{array} \mid\right),
\end{aligned}
$$

where $e_{I, k}^{(0)}$ (or $e_{Q, k}^{(0)}$ ) equals one when there is a decision error and zero when the decision is correct. Thus, $\mathrm{e}^{(0)}=\left(e_{I, 0}^{(0)}, e_{Q, 0}^{(0)}, \cdots, e_{I, K_{C}}^{(0)}, e_{Q, K_{C}}^{(0)}\right)$ represents a set of vectors of $2 K_{C}+2$ data decision errors at the $0^{\text {th }}$ step. Assume that the $N_{e, 0}=N_{e, 0, I}+N_{e, 0, Q}$ bit errors in $\mathrm{e}^{(0)}$ can be divided into $N_{e, 0, I}$ and $N_{e, 0, Q}$ errors in real and imaginary parts, respectively, then the numbers of different error sequences in real and imaginary parts are $\left(\begin{array}{l}K_{C}+1 \\ N_{e, 0, I}\end{array}\right)$ and $\left(\begin{array}{c}K_{C}+1 \\ N_{e, 0, Q}\end{array}\right)$, respectively, where $\left(\begin{array}{c}n \\ k\end{array}\right)=\frac{n !}{k !(n-k) !}$. Thus, $e_{N_{e, 0, I}, i}^{(0)}=\left(e_{I, 0}^{(0)}, \cdots, e_{I, K_{C}}^{(0)}\right)_{N_{e, 0, I}, i}$ is defined as the $i^{\text {th }}\left(i=0,1, \cdots,\left(\begin{array}{c}K_{C}+1 \\ N_{e, 0, I}\end{array}\right)-1\right)$ error sequence for the real part and $e_{N_{e, 0, Q}, j}^{(0)}=\left(e_{Q, 0}^{(0)}, \cdots, e_{Q, K_{C}}^{(0)}\right)_{N_{e, 0, Q}, j}$ is the $j^{\text {th }}\left(j=0,1, \cdots,\left(\begin{array}{c}K_{C}+1 \\ N_{e, 0, Q}\end{array}\right)-1\right)$ error sequence for the imaginary part. $\operatorname{Pr}\left(N_{e, 0} \mid \widehat{\mathrm{H}}_{F}\right)$ can be expressed in terms of $e_{N_{e, 0, I}, i}^{(0)}$ and $e_{N_{e, 0, Q}, j}^{(0)}$, as shown in (15), where the real and imaginary parts are independent to each other, and $\operatorname{Pr}\left(e_{I, 0}^{(0)}, \cdots, e_{I, K_{C}}^{(0)} \mid \widehat{\mathrm{H}}_{F}, N_{e, 0, I}, i\right)$ and $\operatorname{Pr}\left(e_{Q, 0}^{(0)}, \cdots, e_{Q, K_{C}}^{(0)} \mid \widehat{\mathrm{H}}_{F}, N_{e, 0, Q}, j\right)$ are joint event probabilities. Given $N_{e, 0, I}$, it is reasonable to assume that the 


$$
\begin{aligned}
& \operatorname{Pr}\left(N_{e, 0} \mid \widehat{\mathrm{H}}_{F}\right)=\sum_{N_{e, 0, I}=0}^{N_{e, 0}} \sum_{i=0}^{\left(\begin{array}{c}
K_{C}+1 \\
N_{e, 0, I}
\end{array}\right)-1} \sum_{j=0}^{\left(\begin{array}{c}
K_{C}+1 \\
N_{e, 0, Q}
\end{array}\right)-1} \operatorname{Pr}\left(e_{N_{e, 0, I}, i}^{(0)}, e_{N_{e, 0, Q}, j}^{(0)} \mid \widehat{\mathrm{H}}_{F}\right) \\
& =\sum_{N_{e, 0, I}=0}^{N_{e, 0}} \sum_{i=0}^{\left(\begin{array}{c}
K_{C}+1 \\
N_{e, 0, I}
\end{array}\right)-1} \sum_{j=0}^{\left(\begin{array}{l}
K_{C}+1 \\
N_{e, 0}, Q
\end{array}\right)-1} \operatorname{Pr}\left(e_{I, 0}^{(0)}, \cdots, e_{I, K_{C}}^{(0)} \mid \widehat{\mathrm{H}}_{F}, N_{e, 0, I}, i\right) \operatorname{Pr}\left(e_{Q, 0}^{(0)}, \cdots, e_{Q, K_{C}}^{(0)} \mid \widehat{\mathrm{H}}_{F}, N_{e, 0, Q}, j\right) \text {, } \\
& \left\{\begin{array}{l}
\alpha_{M C I}\left(k_{0}, k_{1}\right)=E\left\{M C I_{I, n_{t}-1, k_{0}} \cdot M C I_{I, n_{t}-1, k_{1}}\right\}=\frac{P_{d} \lambda_{0}^{2}}{2} \sum_{m_{0}} \sum_{m_{1}}\left(\begin{array}{l}
\omega_{0, m_{0}} \omega_{0, m_{1}} \widehat{r}_{m_{0}, n_{t}-1, n_{t}-1} \widehat{r}_{m_{1}, n_{t}-1, n_{t}-1}^{\left(k_{0}\right)} c_{N_{F}, m_{0}}^{\left(k_{1}\right)} c_{N_{F}, m_{1}} \sum_{k \neq k_{0}, k_{1}} c_{N_{F}, m_{0}}^{(k)} c_{N_{F}, m_{1}}^{(k)}
\end{array}\right) \\
\alpha_{N o i}\left(k_{0}, k_{1}\right)=E\left\{N o i_{I, n_{t}-1, k_{0}} \cdot N o i_{I, n_{t}-1, k_{1}}\right\}=\frac{\sigma^{2}}{2} \lambda_{0}^{2} \sum_{\bar{m}} \omega_{0, \bar{m}}^{2} c_{N_{F}, \bar{m}}^{\left(k_{0}\right)} c_{N_{F}, \bar{m}}^{\left(k_{1}\right)}
\end{array}\right. \\
& \operatorname{Pr}\left(e_{I, 0}^{(0)}, \cdots, e_{I, K_{C}}^{(0)} \mid \widehat{\mathrm{H}}_{F}, N_{e, 0}, i_{I}\right) \approx \prod_{i=0}^{\left(K_{C}+1\right) / N_{E}-1} \operatorname{Pr}\left(e_{I, i \cdot N_{E}}^{(0)}, e_{I, i \cdot N_{E}+1}^{(0)}, \cdots, e_{I,(i+1) \cdot N_{E}-1}^{(0)} \mid \widehat{\mathrm{H}}_{F}, N_{e, 0}, i_{I}\right)
\end{aligned}
$$

$\operatorname{Pr}\left(e_{I, 0}^{(0)}, \cdots, e_{I, K_{C}}^{(0)} \mid \widehat{\mathrm{H}}_{F}, N_{e, 0, I}, i\right)$ is almost the same for all $i$. Therefore, $\operatorname{Pr}\left(N_{e, 0} \mid \widehat{\mathrm{H}}_{F}\right)$ can be approximated by

$$
\begin{aligned}
& \operatorname{Pr}\left(N_{e, 0} \mid \widehat{\mathrm{H}}_{F}\right) \\
& \approx \sum_{N_{e, 0, I}=0}^{N_{e, 0}}\left[\begin{array}{l}
\left(\begin{array}{c}
K_{C}+1 \\
N_{e, 0, I}
\end{array}\right)\left(\begin{array}{c}
K_{C}+1 \\
N_{e, 0, Q}
\end{array}\right) \\
\cdot \operatorname{Pr}\left(e_{I, 0}^{(0)}, \cdots, e_{I, K_{C}}^{(0)} \mid \widehat{\mathrm{H}}_{F}, N_{e, 0, I}, i_{I}\right) \\
\cdot \operatorname{Pr}\left(e_{Q, 0}^{(0)}, \cdots, e_{Q, K_{C}}^{(0)} \mid \widehat{\mathrm{H}}_{F}, N_{e, 0, Q}, j_{Q}\right)
\end{array}\right]
\end{aligned}
$$

where $i_{I}$ and $j_{Q}$ can be any value chosen from $\left[0,\left(\begin{array}{l}K_{C}+1 \\ N_{e, 0, I}\end{array}\right)-1\right]$ and $\left[0,\left(\begin{array}{c}K_{C}+1 \\ N_{e, 0, Q}\end{array}\right)-1\right]$, respectively. A simple method to calculate $\operatorname{Pr}\left(e_{I, 0}^{(0)}, \cdots, e_{I, K_{C}}^{(0)} \mid \widehat{\mathrm{H}}_{F}, N_{e, 0, I}, i_{I}\right)$ is to assume that the bit error occurring on each code channels is independent to each other, given by

$$
\begin{aligned}
& \operatorname{Pr}\left(e_{I, 0}^{(0)}, \cdots, e_{I, K_{C}}^{(0)} \mid \widehat{\mathrm{H}}_{F}, N_{e, 0, I}, i_{I}\right) \\
& \approx \prod_{k=0}^{K_{C}} \operatorname{Pr}\left(e_{I, k}^{(0)} \mid \widehat{\mathrm{H}}_{F}, N_{e, 0, I}, i_{I}\right)
\end{aligned}
$$

where $\operatorname{Pr}\left(e_{I, k}^{(0)} \mid \widehat{\mathrm{H}}_{F}, N_{e, 0, I}, i_{I}\right)=P_{e, \widehat{\mathrm{H}}_{F}}^{(0)}$ for $e_{I, k}^{(0)}=1$, and $\operatorname{Pr}\left(e_{I, k}^{(0)} \mid \widehat{\mathrm{H}}_{F}, N_{e, 0, I}, i_{I}\right)=1-P_{e, \widehat{\mathrm{H}}_{F}}^{(0)}$ for $e_{I, k}^{(0)}=0$. However, it is noted that for different code channels at the same step of the ZF-MMSE-SIC detection, the MCI (or noise) terms in the decision variable of real part (or imaginary part) are correlated to each other, as shown in (18). Therefore, the decision variables on the $K_{C}+1$ code channels are correlated and the calculation of $\operatorname{Pr}\left(e_{I, 0}^{(0)}, \cdots, e_{I, K_{C}}^{(0)} \mid \widehat{\mathrm{H}}_{F}, N_{e, 0, I}, i_{I}\right)$ involves a multivariate Gaussian distribution of $K_{C}+1$ dimensions, which becomes intractable when $K_{C}+1$ is large. Assume that $\operatorname{Pr}\left(e_{I, 0}^{(0)}, \cdots, e_{I, K_{C}}^{(0)} \mid \widehat{\mathrm{H}}_{F}, N_{e, 0, I}, i_{I}\right)$ can be approximated by a set of $N_{E}$-event probabilities, given by (19). When $N_{E}=K_{C}+1$, (19) provides the accurate value at the cost of very high complexity. As $N_{E}$ decreases, the value of (19) deviates from the accurate one since more and more correlations are ignored, but the complexity is also reduced. By setting $N_{E}=1$, (19) reduces to (17) and the calculation becomes simple. However, since no correlation is considered when $N_{E}=1$, the approximation is poor. It is noted that although the two-event probability with $N_{E}=2$ needs more effort to calculate than that with $N_{E}=1$, it can be upper bounded by a close form equation, which reduces the complexity. By taking some level of correlation into account, the approximation using $N_{E}=2$ should be more accurate than that provided by $N_{E}=1$, which will be verified by simulations in next section. Moreover, although the correlations between the two-events are ignored, it will be shown later that the analyzed BERs based on the twoevent approximation are close to the simulated ones and provides a good estimation. Thus, $N_{E}$ is set to two in this letter. When there are two decision errors, the probability $\operatorname{Pr}\left(e_{I, 0}^{(0)}, e_{I, 1}^{(0)} \mid \widehat{\mathrm{H}}_{F}, N_{e, 0}, i_{I}\right)$ can be upper bounded by [7]

$$
\begin{aligned}
& \operatorname{Pr}\left(e_{I, 0}^{(0)}=1, e_{I, 1}^{(0)}=1 \mid \widehat{\mathrm{H}}_{F}\right) \\
& =\operatorname{Pr}\left(d_{I, 0,0} y_{I, 0,0} \leq 0, d_{I, 0,1} y_{I, 0,1} \leq 0 \mid \widehat{\mathrm{H}}_{F}\right) \\
& \leq \frac{1}{2} \frac{\sqrt{1-\alpha_{0,1}}}{1-\alpha_{0,1}^{(1)}}\left[Q\left(\sqrt{\frac{P_{d}\left(1-\alpha_{0,1}^{(1)}\right)}{2 \sigma_{I}^{2}(0)}}\right)\right]^{2} \\
& \quad+\frac{1}{2} \frac{\sqrt{1-\alpha_{0,1}}}{1+\alpha_{0,1}^{(2)}}\left[Q\left(\sqrt{\frac{P_{d}\left(1+\alpha_{0,1}^{(2)}\right)}{2 \sigma_{I}^{2}(0)}}\right)\right]^{2}
\end{aligned}
$$

where $\alpha_{0,1}=\alpha_{M C I}(0,1)+\alpha_{N o i}(0,1), \alpha_{0,1}^{(1)}=\max \left(0, \alpha_{0,1}\right)$ and $\alpha_{0,1}^{(2)}=\min \left(0, \alpha_{0,1}\right)$. Similarly, when there is one and no decision error, the corresponding event probabilities are given by

$$
\begin{aligned}
& \operatorname{Pr}\left(e_{I, 0}^{(0)}=1, e_{I, 1}^{(0)}=0 \mid \widehat{\mathrm{H}}_{F}\right) \\
& =\operatorname{Pr}\left(e_{I, 0}^{(0)}=0, e_{I, 1}^{(0)}=1 \mid \widehat{\mathrm{H}}_{F}\right) \\
& =\operatorname{Pr}\left(d_{I, 0,0} y_{I, 0,0} \geq 0, d_{I, 0,1} y_{I, 0,1} \leq 0 \mid \widehat{\mathrm{H}}_{F}\right) \\
& \leq Q\left(\sqrt{\frac{P_{d}}{2 \sigma_{I}^{2}(0)}}\right)-\frac{1}{2} \frac{\sqrt{1-\alpha_{0,1}}}{1-\alpha_{0,1}^{(2)}}\left[Q\left(\sqrt{\frac{P_{d}\left(1-\alpha_{0,1}^{(2)}\right)}{2 \sigma_{I}^{2}(0)}}\right)\right]^{2} \\
& -\frac{1}{2} \frac{\sqrt{1-\alpha_{0,1}}}{1+\alpha_{0,1}^{(1)}}\left[Q\left(\sqrt{\frac{P_{d}\left(1+\alpha_{0,1}^{(1)}\right)}{2 \sigma_{I}^{2}(0)}}\right)\right]^{2},
\end{aligned}
$$


TABLE I

SYSTEM PARAMETERS

\begin{tabular}{|c|c|}
\hline No. of transmit antennas $n_{t}$ & 4 \\
\hline No. of receive antennas $n_{r}$ & 4 \\
\hline Bandwidth & $100 \mathrm{MHz}$ \\
\hline No. of sub-carriers $M$ & $\begin{array}{l}1024(97.7 \mathrm{kHz} \text { sub-carrier spac- } \\
\text { ing) }\end{array}$ \\
\hline $\begin{array}{l}\text { OFCDM symbol duration } \\
T_{s}=T_{e}+T_{g}\end{array}$ & $12.5 \mu s=10.24+2.26$ \\
\hline $\begin{array}{l}\text { Packet length }\left(T_{s} \cdot\left(N_{P}+\right.\right. \\
\left.\left.N_{D}\right)\right) \text { (No. of OFCDM sym- } \\
\text { bols per packet) }\end{array}$ & $\begin{array}{l}\mathbf{0 . 6 5 m s}(12.5 \mu s \cdot(4+48))(\mathbf{5 2} \\
\text { OFCDM symbols: } \mathbf{4} \text { for pilot and } \\
\mathbf{4 8} \text { for data) }\end{array}$ \\
\hline Pilot Channel & $\begin{array}{l}\text { Time-multiplexed with data chan- } \\
\text { nels one dimensional spreading in } \\
\text { the time domain }\end{array}$ \\
\hline $\begin{array}{l}\text { Spreading Code for Data } \\
\text { Channel }\end{array}$ & $\begin{array}{l}\text { 2-D OVSF (spreading factor } N= \\
N_{T} \times N_{F}=8 \times N_{F} \text { ) }\end{array}$ \\
\hline $\begin{array}{l}\text { Spreading Code for Pilot } \\
\text { Channel }\end{array}$ & OVSF (spreading factor $N_{P}=4$ ) \\
\hline System Load & $K / N=1.0$ \\
\hline Modulation & $\begin{array}{l}\text { QPSK for both data and pilot } \\
\text { channels }\end{array}$ \\
\hline Channel Model & $\begin{array}{l}\text { Parallel slow fading multi- } \\
\text { channel model with a coherence } \\
\text { bandwidth of } \Delta f_{c}=1 \mathrm{MHz}\end{array}$ \\
\hline Channel Estimation & $\begin{array}{l}\text { Pilot-aided, ZF channel estima- } \\
\text { tion }\end{array}$ \\
\hline Signal Detection & ZF-MMSE-SIC \\
\hline
\end{tabular}

and

$$
\begin{aligned}
& \operatorname{Pr}\left(e_{I, 0}^{(0)}=0, e_{I, 1}^{(0)}=0 \mid \widehat{\mathrm{H}}_{F}\right) \\
& =\operatorname{Pr}\left(d_{I, 0,0} y_{I, 0,0} \geq 0, d_{I, 0,1} y_{I, 0,1} \geq 0 \mid \widehat{\mathrm{H}}_{F}\right) \\
& \leq 1+\frac{1}{2} \frac{\sqrt{1-\alpha_{0,1}}}{1-\alpha_{0,1}^{(1)}}\left[Q\left(\sqrt{\frac{P_{d}\left(1-\alpha_{0,1}^{(1)}\right)}{2 \sigma_{I}^{2}(0)}}\right)\right]^{2} \\
& +\frac{1}{2} \frac{\sqrt{1-\alpha_{0,1}}}{1+\alpha_{0,1}^{(2)}}\left[Q\left(\sqrt{\frac{P_{d}\left(1+\alpha_{0,1}^{(2)}\right)}{2 \sigma_{I}^{2}(0)}}\right)\right]^{2}-2 Q\left(\sqrt{\frac{P_{d}}{2 \sigma_{I}^{2}(0)}}\right),
\end{aligned}
$$

respectively. Using (16), (19) and the bounds (20)-(22), an approximation of $\operatorname{Pr}\left(N_{e, 0} \mid \widehat{\mathrm{H}}_{F}\right)$ is obtained.

In (13), the calculations of $\operatorname{Pr}\left(N_{e, 1} \mid N_{e, 0}, \widehat{\mathrm{H}}_{F}\right), \cdots$, and $\operatorname{Pr}\left(N_{e, s-1} \mid N_{e, 0}, \cdots, N_{e, s-2}, \widehat{\mathrm{H}}_{F}\right)$ can be carried out in a similar way as that of $\operatorname{Pr}\left(N_{e, 0} \mid \widehat{\mathrm{H}}_{F}\right)$, by taking the MAI in the decision variable as a Gaussian variable. Moreover, it is observed in the numerical results that when the number of bit errors is larger than 8 , the contribution to $P_{e, \widehat{\mathrm{H}}_{F}}^{(s)}$ from corresponding items in (10) is negligible. Therefore, no more than 8 bit errors are taken into account when calculating $P_{e, \widehat{\mathrm{H}}_{F}}^{(s)}$. Finally, $P_{e, \widehat{\mathrm{H}}_{F}}^{(s)}$ is averaged over all $\widehat{\mathrm{H}}_{F}$ to obtain the final average BER at the $s^{\text {th }}$ step, which can be numerically evaluated by a Monte Carlo Approach. Although the BER analysis is carried out for QPSK, the same approach can be extended to other rectangular QAM schemes with a more complex error enumerating process.

\section{Performance Evaluation}

Some representative numerical and simulation results are shown in this section. The configuration of the multi-code
MIMO-OFCDM system is given in Table I. Unless noted otherwise, the power ratio between the pilot channel and all data channels $\beta / K$ is set to 3.5. First of all, given four transmit and receive antennas, the BER performance of the transceiver A (with multi-code transmission and ZF-MMSE-SIC) and B (with orthogonal single-code transmission) is compared in Fig. 2(a) by means of simulations. It can be seen that in terms of BER, transceiver B outperforms transceiver A due to the orthogonal transmission. However, the effective data rate provided by transceiver $\mathrm{B}$ is only $1 / N_{T, B}=1 / 4$ as high as that provided by transceiver $A$. In the following investigations, transceiver $\mathrm{A}$ is considered.

The analytical performance of an uncoded system is verified by simulations in Fig. 2 as a function of SNR. As a comparison, Fig. 2(a) and 2(b) plot the BERs calculated from the proposed analytical approach (approach (a)) and the one where no correlation is considered (using (17), approach (b)), respectively. It can be seen that for the $3^{\text {rd }}$ antenna, the analytical results of both approaches match well with the simulated ones, because at the $0^{\text {th }}$ step of ZF-MMSE-SIC detection there is no MAI cancellation and no error propagation. For later detected antennas, the analytical results of approach (a) keep close to the simulated BER, while the results of approach (b) deviate from the simulated ones, especially at high SNR. The reason is that as the ZF-MMSE-SIC detection goes on step by step, the errors at previous steps propagate to the current one and have a cumulative effect on the BER performance. Their influence is apparent in the $2^{\text {nd }}$ and $3^{\text {rd }}$ steps as they dominate the BER performance, particularly at high SNR. It is clear that the correlation between the error events at previous steps should be taken into account, otherwise the analytical BER becomes too optimistic.

As illustrated in Section II, the MIMO-OFCDM system employs a practical channel estimation algorithm by using the time-multiplexed pilot channel. Since the total transmission power is limited, the power should be suitably allocated to the data channels and the pilot channel to achieve the best performance. Fig. 3 shows the system performance as a function of the power ratio between the pilot and all data channels $(\beta / K)$. It can be seen that when $\beta / K$ is small, the pilots have low power and the channel estimation quality is poor, resulting in a high BER. When $\beta / K$ increases, the BER reduces as the quality of channel estimation improves. The BER reaches a minimum value for a particular value of $\beta / K$. Further increasing $\beta / K$ beyond that value increases BER due to too little power assigned to data channels. At different antennas, the system always achieves near optimum performance when $\beta / K$ takes value between 2.0 to 6.0 . It can also be seen that the analytical results obtained from the proposed approach matches well with the simulations for all values of $\beta / K$, while the analytical BER of approach (b) for the last stage of detection is much lower than the simulated BER, especially near the optimum $\beta / K$. This again demonstrates the importance of taking correlation into consideration in order to obtain accurate performance evaluation. Note that for the sake of clarity, the analytical results of approach (b) are only shown for the last stage of detection.

Finally, the BER performance is shown in Fig. 4 as a function of frequency domain spreading factor $N_{F}$ when $N_{T}$ 


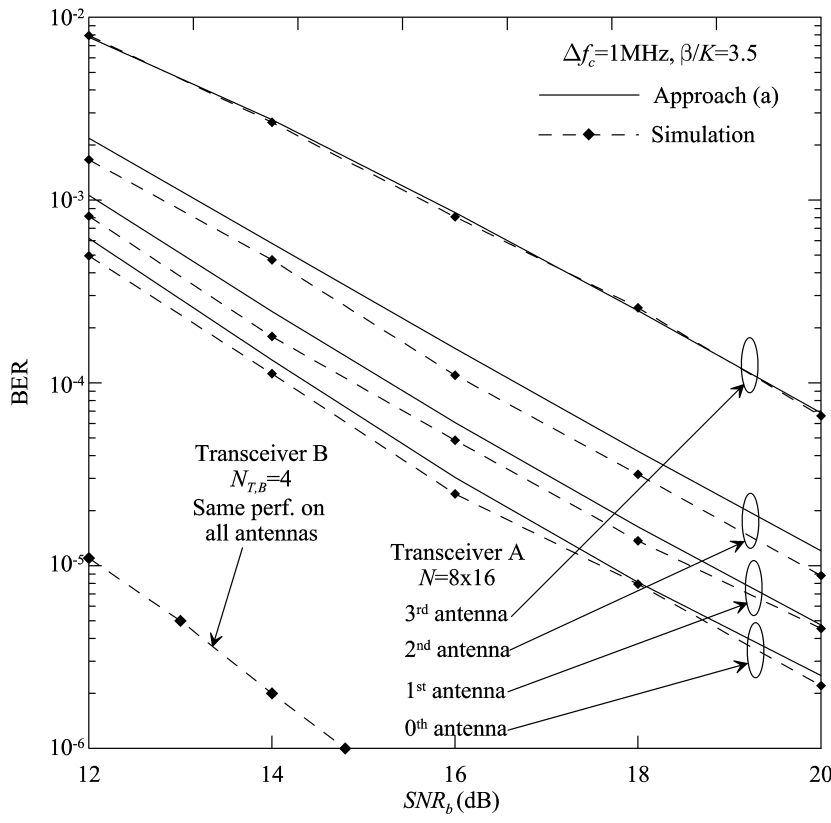

Figure 2a. System performance as a function of $S N R_{b}$

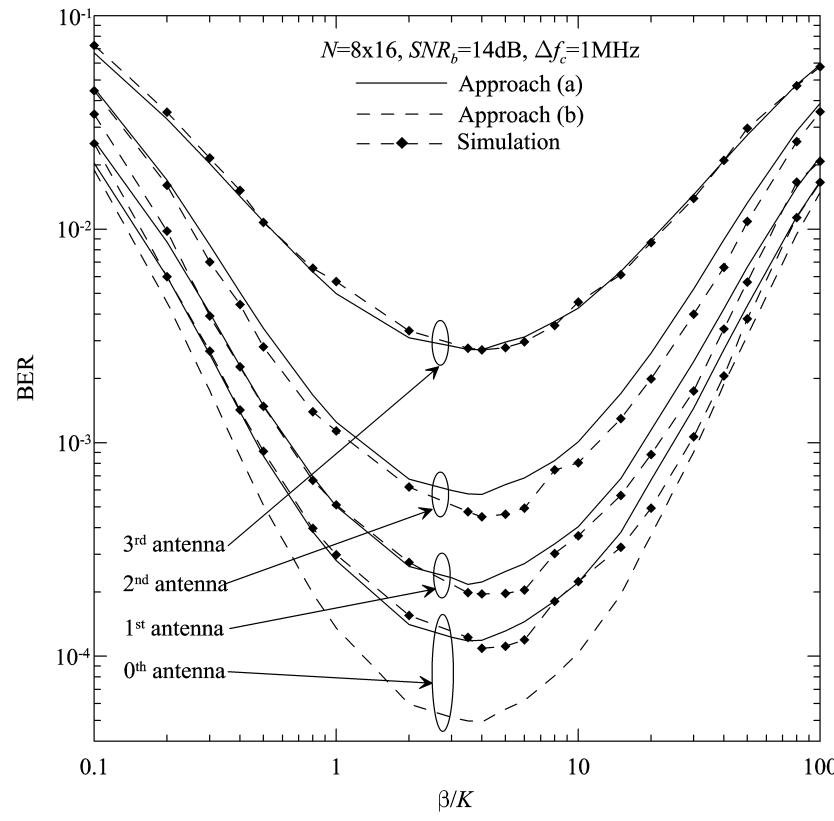

Fig. 3. System performance as a function of power ratio

is fixed at eight. For various values of $N_{F}$, the analytical results of approach (a) are accurate. On the other hand, only for small $N_{F}$ like $N_{F} \leq 4$, the BER of approach (b) is close to the simulated BER at the last stage of detection. When $N_{F}$ gets larger, it deviates further and further from the simulation result. Since the number of code channels increases with $N_{F}\left(K_{C}=N_{F}-1\right)$, more error events occur at each step of ZF-MMSE-SIC detection. Thus the error event correlation has greater influence on the system performance with larger $N_{F}$. Moreover, it can be seen that when $N_{F}$ increases, the performance of MIMO-OFCDM improves, especially for the $2^{\text {nd }}, 1^{\text {st }}$, and $0^{\text {th }}$ antennas. This is in consistence with that in [1].

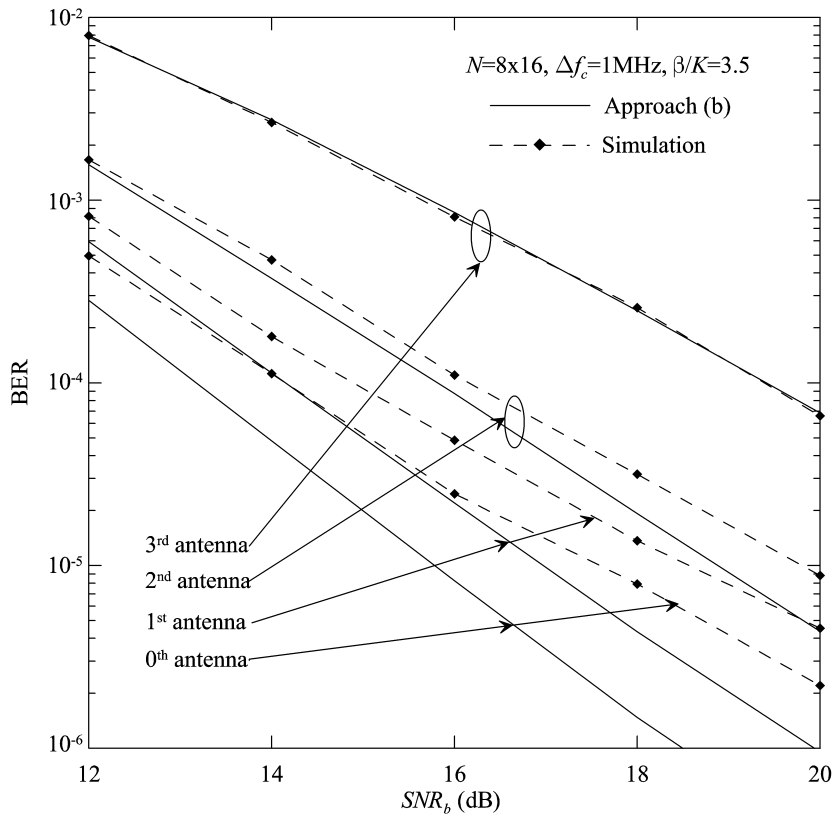

Figure 2b. System performance as a function of $S N R_{b}$

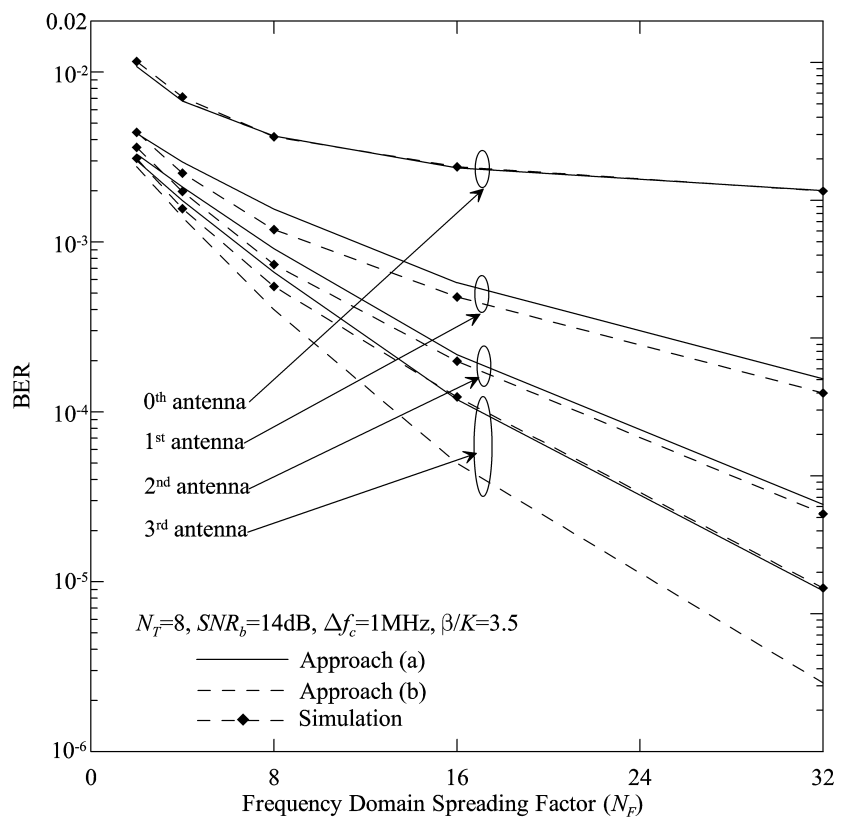

Fig. 4. Effect of frequency domain spreading factor

\section{CONCLUSION}

This letter has proposed an analytical approach for the performance evaluation of MIMO-OFCDM with ZF-MMSESIC. The analytical results have been verified by simulations with various channel conditions. It has been shown that in MIMO-OFCDM systems with multi-code transmission, at each step of SIC, the error events on multiple code channels are correlated to each other. By taking the correlation between error events into account, the proposed analytical approach provides a good estimation for the system performance with various channel conditions, which is more accurate than the one where no correlation is considered. 


\section{ACKNOWLEDGMENT}

This work was supported by The University of Hong Kong Research Committee.

\section{REFERENCES}

[1] Y. Q. Zhou and T. S. Ng, "MIMO-OFCDM systems with joint iterative detection and optimal power allocation," IEEE Trans. Wireless Commun., pp. 5504-5516, Dec. 2008.

[2] H. Atarashi, S. Abeta, and M. Sawahashi, "Broadband packet wireless access appropriate for high-speed and high-capacity throughput," in Proc. IEEE VTC2001-Spring, pp. 566-570, May 2001

[3] W. Yin, C. C. Tsimenidis, and B. S. Sharif, "Transmission power optimization of convolutional coded VBLAST system," in Proc. IEEE VTC2005-Spring, pp. 945-948, June 2005.
[4] K. Higuchi, H. Kawai, N. Maeda, and M. Sawahashi, "Adaptive selection of surviving symbol replica candidates based on maximum reliability in QRM-MLD for OFCDM MIMO multiplexing," in Proc. IEEE Globecom 2004, pp. 2480-2486, Nov. 2004.

[5] S. Loyka and F. Gagnon, "Performance analysis of the V-BLAST algorithm: an analytical approach," IEEE Trans. Wireless Commun., vol. 3, pp. 1326-1337, July 2004.

[6] M. K. Varanasi and B. Aazhang, "Multistage detection in asynchronous code-division multiple-access communications," IEEE Trans. Commun., vol. 38, no. 4, pp. 509-519, Apr. 1990.

[7] A. H. Madsen and K. S. Cho, "MMSE/PIC multiuser detection for DS/CDMA systems with inter-and intra-cell interference," IEEE Trans. Commun., vol. 47, pp. 291-299, Feb. 1999.

[8] W. C. Y. Lee, Mobile Communications Engineering: Theory and Applications, 2nd ed. New York: McGraw-Hill, 1997. 